\title{
Fourier Transform Light Scattering of Biological Structure and Dynamics
}

\author{
Huafeng Ding, Ethan Berl, Zhuo Wang, Larry J. Millet, Martha U. Gillette, Jianming Liu, Marni Boppart, \\ and Gabriel Popescu
}

(Invited Paper)

\begin{abstract}
We review the principle and application of Fourier transform light scattering (FTLS), a new technique developed in our laboratory to study static and dynamic light scattering (DLS) from the biological tissues and live cells. The results demonstrate that FTLS has significant benefits over existing light scattering techniques in terms of sensitivity and resolution. We anticipate that FTLS will set the basis for disease diagnosis based on the intrinsic tissue optical properties and provide an efficient tool for quantifying cell structures and dynamics.
\end{abstract}

Index Terms-Cells, Fourier transform light scattering (FTLS), light scattering, phase, quantitative phase imaging (QPI), tissue.

\section{INTRODUCTION}

$\mathbf{L}$ IGHT scattering has been widely used for studying biological samples, as it is noninvasive, requires minimum sample preparation, and extracts rich information about morphology and dynamic activity [1]-[9]. Applying dynamic light scattering (DLS) measurements to monitor the dynamic changes inside biological samples has emerged as an important approach in the field [10]-[14]. More recently, efforts have been devoted to study the mechanical properties of a complex fluid by detecting dynamic scattering from probing particles [15]-[24]. Thus, the viscoelastic properties of complex fluids are retrieved over various temporal scales. This method was further extended to the cell membranes with attached micron-sized beads as probes. This provides a new way to study the microrheology of live cells [25]. The information is extracted from the temporal light scattering signals associated with the activity of individual cell component such as filamentous actin and microtubules. The polymerization and depolymerization of these cytoskeleton structures are highly dynamic processes and have important functional roles [26].

Manuscript received August 2, 2009; revised September 15, 2009; accepted September 15, 2009 . Date of publication April 5, 2010; date of current version August 6, 2010. The work of G. Popescu was supported in part by the National Science Foundation (CAREER 08-46660) and Grainger Foundation.

H. Ding, E. Berl, Z. Wang, and G. Popescu are with the Quantitative Light Imaging Laboratory, Department of Electrical and Computer Engineering, Beckman Institute for Advanced Science and Technology, University of Illinois at Urbana-Champaign, Urbana, IL 61801 USA (e-mail: ding@illinois.edu; ethanberl@gmail.com; wangzhs@gmail.com; gpopescu@illinois.edu).

L. J. Millet and M. U. Gillette are with the Department of Cell and Developmental Biology, University of Illinois at Urbana-Champaign, Urbana, IL 61801 USA (e-mail: millet@life.uiuc.edu; mgillette@illinois.edu).

J. Liu and M. Boppart are with the Department of Kinesiology and Community Health, Beckman Institute for Advanced Science and Technology, University of Illinois at Urbana-Champaign, Urbana, IL 61801 USA (e-mail: liu10uiuc@gmail.com; mboppart@illinois.edu).

Color versions of one or more of the figures in this paper are available online at http://ieeexplore.ieee.org.

Digital Object Identifier 10.1109/JSTQE.2009.2034752

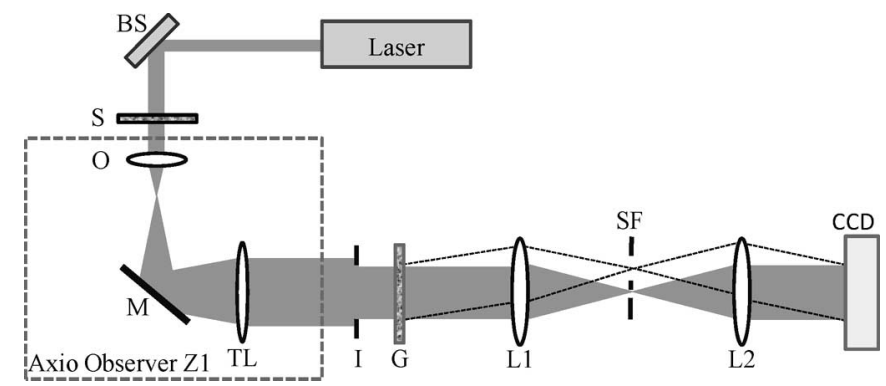

Fig. 1. FTLS experimental setup. BS, beam splitter; S, sample; O, objective lens; M, mirror; TL, tube lens; I, iris; G, Grating; SF, spatial filter; and L1 and L2, lenses. Figure adapted from [39].

Light scattering techniques provide information that is intrinsically averaged over the measurement volume. Thus, the spatial resolution is compromised and the scattering contributions from individual components are averaged. Particle tracking microrheology has recently been proposed to measure the particle displacements in the imaging (rather than scattering) plane [27] in which the spatial resolution is reserved. However, the drawback is that relatively large particles are needed such that they can be tracked individually, which also limits the throughput required for significant statistical average. Recently, phase-sensitive methods have been employed to directly extract the refractive index of cells and tissues [28], [29]. From this information, the angular scattering can be achieved via the Born approximation [30].

Based on diffraction phase microscopy (DPM) [31], we developed Fourier transform light scattering (FTLS) as an approach to study static and DLS. FTLS combines the high spatial resolution associated with optical microscopy and intrinsic averaging of light scattering techniques [32]. Due to its common path interferometric geometry, DPM is stable in optical path length to the subnanometer level. This feature allows FTLS to perform studies on static and dynamic samples with unprecedented sensitivity. In this paper, we describe the principle of FTLS and review the application of FTLS to study both static and DLS from biological tissues and live cells.

\section{PRINCIPLE OF FTLS}

As described in [32], our FTLS system requires accurate phase retrieval for elastic light scattering (ELS) measurements and in addition, fast acquisition speed for DLS studies. Fig. 1 depicts our experimental setup that satisfies these requirements 
by incorporating a common path interferometer with a commercial computer-controlled microscope. The second harmonic of an Nd:YAG laser $(\lambda=532 \mathrm{~nm})$ is used to illuminate the sample in transmission. To ensure full spatial coherence, the laser beam is coupled into a single-mode fiber and further collimated by a fiber collimator. The light scattered by the sample is collected by the objective lens of the microscope (AxioObserver Z1, Zeiss) and imaged at the side port of the microscope. A diffraction grating $\mathrm{G}$ is placed at this image plane, thus generating multiple diffraction orders containing full spatial information about the image. In order to establish a common path Mach-Zehnder interferometer, a standard spatial filtering lens system $\mathrm{L}_{1}-\mathrm{L}_{2}$ is used to select the two diffraction orders and generate the final interferogram at the CCD plane. The zeroth-order beam is lowpass filtered using the spatial filter SF positioned in the Fourier plane of $\mathrm{L}_{1}$ such that at the CCD plane, it approaches a uniform field. Simultaneously, the spatial filter allows passing the entire frequency content of the first diffraction order beam and blocks all the other orders. The first order is thus the imaging field and the zeroth order plays the role of the reference field. The two beams propagate along a common optical path, thus significantly reducing the longitudinal phase noise. The direction of the spatial modulation is along the $x$-axis such that the total field at the CCD plane has the form:

$$
U(x, y)=\left|U_{0}\right| e^{i\left(\phi_{0}+\beta x\right)}+\left|U_{1}(x, y)\right| e^{i \phi_{1}(x, y)} .
$$

In (1), $\left|U_{0,1}\right|$ and $\phi_{0,1}$ are the amplitudes and the phases of the orders of diffraction 0,1 , while $\beta$ represents the spatial frequency shift induced by the grating to the zeroth order. To preserve the transverse resolution of the microscope, the spatial frequency $\beta$ exceeds the maximum frequency allowed by the numerical aperture of the instrument. The $\mathrm{L}_{1}-\mathrm{L}_{2}$ lens system has an additional magnification of $f_{2} / f_{1}=5$ such that the sinusoidal modulation of the image is sampled by four charge-coupled device (CCD) pixels per period. The interferogram is spatially high-pass filtered to isolate the cross term:

$$
\left|U_{0}\right|\left|U_{1}(x, y)\right| \cos \left[\phi_{1}(x, y)-\phi_{0}-\beta x\right]
$$

which can be regarded as the real part of a complex analytic signal. The imaginary component $\sin \left[\phi(x, y)-\phi_{0}-\beta x\right]$ is obtained via a spatial Hilbert transform [31], [33], [34]. Thus, from a single CCD exposure, we obtain the spatially resolved phase and amplitude associated with the image field. From this image field information $\widetilde{U}$, the complex field can be numerically propagated at arbitrary planes; in particular, the far-field angular scattering distribution $\widetilde{U}$ can be obtained simply via a Fourier transformation [32]:

$$
\widetilde{U}(\mathbf{q}, t)=\int U(\mathbf{r}, t) e^{-i \mathbf{q} \cdot \mathbf{r}} d^{2} \mathbf{r} .
$$

With time-lapse image acquisition, the temporal scattering signals are recorded and the sampling frequency is only limited by the speed of the camera. The power spectrum is obtained through Fourier transform of these time-resolved scattering signals.

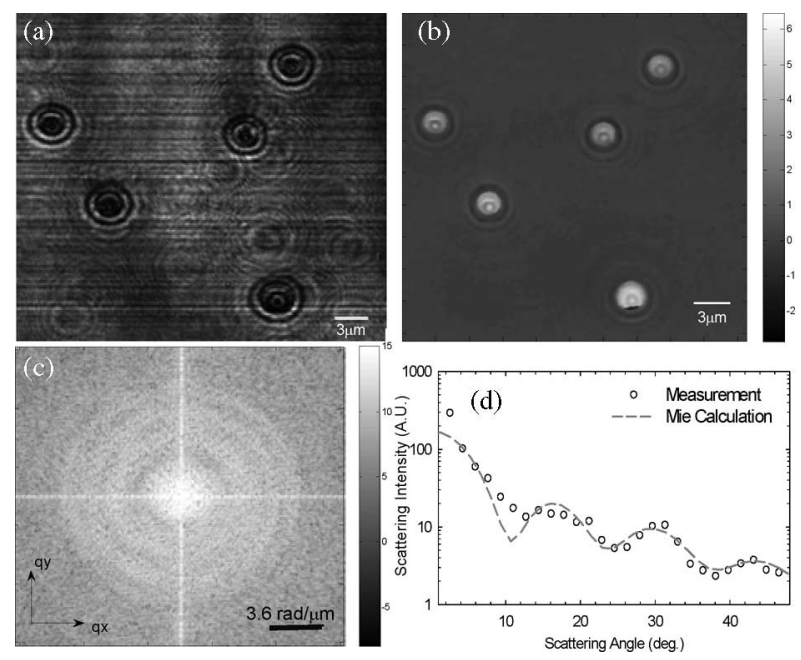

Fig. 2. FTLS reconstruction procedure of angular scattering from $3 \mu \mathrm{m}$ beads. (a) Amplitude image. (b) Reconstructed phase image. (c) Scattering wave vector map. (d) Retrieved angular scattering and comparison with Mie calculation. Figure adapted from [32].

As the system calibration, we applied FTLS to dilute microsphere water suspensions sandwiched between two cover slips, the scattering of which can be easily modeled with Mie calculation as shown in [32]. The measured complex field associated with such samples can be expressed as [32]

$$
U(\mathbf{r} ; t)=\iint_{A} U_{F}\left(\mathbf{r}^{\prime}\right) \sum_{i=1}^{N} \delta\left\{\left[\mathbf{r}-\mathbf{r}_{i}(t)\right]-\mathbf{r}^{\prime}\right\} d^{2} \mathbf{r}^{\prime} .
$$

In (4), $U_{F}$ is the (time invariant) complex field associated with each particle, $\delta$ is the 2-D Dirac function describing the position $\left(x_{i}, y_{i}\right)$ of each of the $N$ moving particles, and the integral is performed over the microscope field of view A.

Fig. 2(a) and (b) shows the amplitude and phase distributions obtained by imaging $3 \mu \mathrm{m}$ polystyrene beads at a particular point in time. For ELS studies, prior to processing the interferogram, we subtract a background image obtained as the intensity map without sample or reference beam. The scattered far-field is obtained by Fourier transforming (4) in space. This angular field distribution factorizes into a form field $\widetilde{U}$, which is determined by the angular scattering of a single particle, and a structure field $\widetilde{U}$, describing the spatial correlations in particle positions [32]:

$$
\widetilde{U}(\mathbf{q} ; t)=\widetilde{U}_{F}(\mathbf{q}) \widetilde{U}_{S}(\mathbf{q} ; t)
$$

where $\mathbf{q}$ is the spatial wave vector and $\widetilde{U}_{S}(\mathbf{q} ; t)=\sum_{i} e^{i \mathbf{q} \cdot \mathbf{r}_{\mathbf{i}}(t)}$. Fig. 2(c) shows the resulting intensity distribution $\left|\widetilde{U}_{F}(\mathbf{q})\right|^{2}$ for the beads in Fig. 2(a) and (b). As expected for such sparse distributions of particles, the form function is dominant over the entire angular range. However, by finding the phase-weighted centroid of each particle, FTLS can retrieve independently the structure function whenever it has a significant contribution to the far-field scattering, e.g., in colloidal crystals. The scattered intensity [e.g., Fig. 2(c)] is averaged over rings of constant wave vectors, $q=(4 \pi / \lambda) \sin (\theta / 2)$, with $\theta$ the scattering angle, as exemplified in Fig. 2(d). In order to test the ability of FTLS 
to retrieve quantitatively the form function of the spherical dielectric particles, we used Mie theory for comparison [35]. The oscillations in the angular scattering establish the quantitative agreement between the FTLS measurement and Mie theory, which contrasts with the common measurements on colloidal suspensions, where the signal is averaged over a large number of scatterers.

\section{FTLS OF TISSUES}

Upon propagation through inhomogeneous media, optical fields suffer modifications in terms of irradiance, phase, spectrum, direction, polarization, and coherence, which can reveal information about the sample of interest. Light scattering by cells and tissues evolved as a dynamic area of study, especially because this type of investigation can potentially offer a noninvasive window into function and pathology [36]-[38].

Recently, we have used FTLS to extract quantitatively the scattering mean free path $l_{s}$ and anisotropy factor $g$ from tissue slices of different organs [39]. This direct measurement of tissue scattering parameters allows predicting the wave transport phenomena within the organ of interest at a multitude of scales. The scattering mean free path $l_{s}$ was measured by quantifying the attenuation due to scattering for each slice via the Lambert-Beer law, $l_{s}=-d / \ln \left[I(d) / I_{0}\right]$, where $d$ is the thickness of the tissue, $I(d)$ is the irradiance of the unscattered light after transmission through the tissue, and $I_{0}$ is the total irradiance, i.e., the sum of the scattered and unscattered components. The unscattered intensity $I(d)$, i.e., the spatial dc component, is evaluated by integrating the angular scattering over the diffraction spot around the origin. The resulting $l_{s}$ values for 20 samples for each organ from the same rat are summarized in Fig. 3(a).

The anisotropy factor $g$ is defined as the average cosine of the scattering angle:

$$
g=\frac{\int_{-1}^{1} \cos (\theta) p[\cos (\theta)] d[\cos (\theta)]}{\int_{-1}^{1} p[\cos (\theta)] d[\cos (\theta)]}
$$

where $p$ is the normalized angular scattering, i.e., the phase function. Note that since (3) applies to tissue slices of thickness $d<l_{s}$, it cannot be used directly in (6) to extract $g$ since $g$ values in this case will be thickness-dependent. This is so because the calculation in (6) is defined over tissue of thickness $d=l_{s}$, which describes the average scattering properties of the tissue (i.e., independent of how the tissue is cut). Under the weakly scattering regime of interest here, this angular scattering distribution $p$ is obtained by propagating the complex field numerically through $N=l_{s} / d$ layers of $d=5 \mu \mathrm{m}$ thickness:

$$
p(\mathbf{q}) \propto\left|\iint[U(\mathbf{r})]^{N} e^{i \mathbf{q} \cdot \mathbf{r}} d^{2} \mathbf{r}\right|^{2} .
$$

Equation (7) applies to a slice of thickness $l_{s}$. It reflects that by propagating through $N$ weakly scattering layers of tissue, the total phase accumulation is the sum of the phase shifts from each layer, as is typically assumed in phase imaging of transparent structures [40]. The angular scattering distribution, or phase function, $p(\theta)$ is obtained by performing azimuthal averaging of the scattering map $p(\mathbf{q})$, associated with each tissue sample.
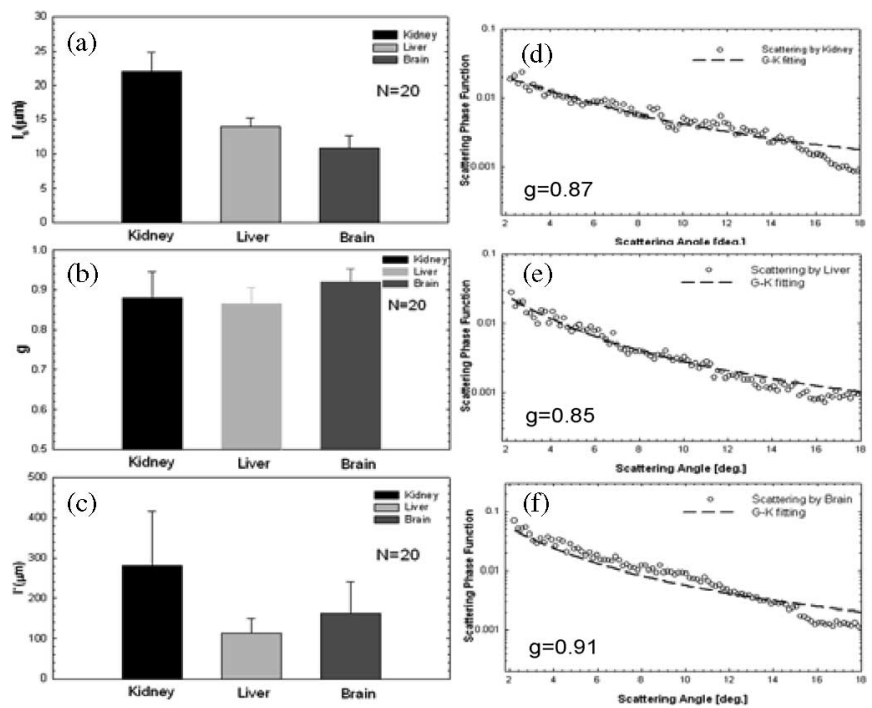

Fig. 3. (a) FTLS measurements of the scattering mean free path $l_{s}$, (b) anisotropy factors, (c) and transport mean free path for the three rat organs with 20 samples per group. The error bars correspond to the standard deviations $(N=20)$. (d)-(f) Angular scattering plots. The dash lines indicate fits with the G-K phase function. Figure adapted from [39].

The maximum scattering angle was determined by the numerical aperture of the objective lens and it is about $18^{\circ}$ for our current setup $(10 \times$ objectives). The angular scattering data were further fitted with Gegenbauer Kernel (GK) phase function [41]

$$
\begin{aligned}
& P(\theta) \\
& =a g \cdot \frac{\left(1-g^{2}\right)^{2 a}}{\pi\left[1+g^{2}-2 g \cos (\theta)\right]^{(a+1)}\left[(1+g)^{2 a}-(1-g)^{2 a}\right]} .
\end{aligned}
$$

Note that $g$ can be estimated directly from the angular scattering data via its definition (6). However, because of the limited angular range measured, $g$ tends to be overestimated by this method, and thus, the GK fit offers a more reliable alternative. The representative fitting plots for each sample are shown in Fig. 3(d) and (f). The final values of $g$ are included in Fig. 3(b) and agree very well with previous reports in the literature [42]. From these measurements of thin, singly scattering slices, we inferred the behavior of light transport in thick, strongly scattering tissue. Thus, the transport mean free path, which is the renormalized scattering length to account for the anisotropic phase function, can be obtained as $l^{*}=l_{s} /(1-g)$. The $l^{*}$ values for 20 samples from each organ are shown in Fig. 3(c).

The results aforementioned showed that FTLS can quantify the angular scattering properties of thin tissues, which thus provides the scattering mean free path $l_{s}$ and anisotropy factor $g$ for the macroscopic (bulk) organ. We note that based on the knowledge of $l_{s}, g$, and $l^{*}$, one can predict the outcome of a broad range of scattering experiments on large samples (size $>>l^{*}$ ), via numerical solutions to the transport equation, or analytical solutions to the diffusion equation [43]. We envision that the FTLS measurements of unstained tissue biopsies, which are broadly available, will not provide only diagnosis value, but 


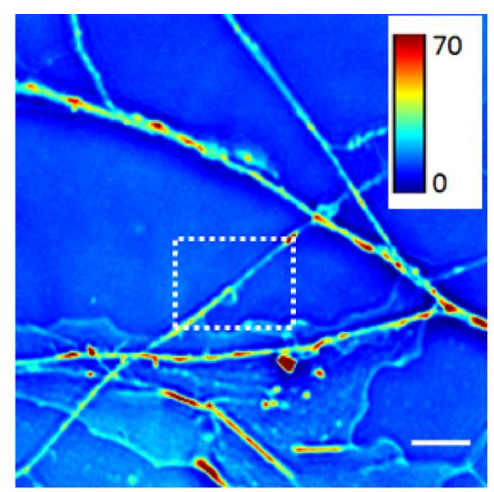

(a)

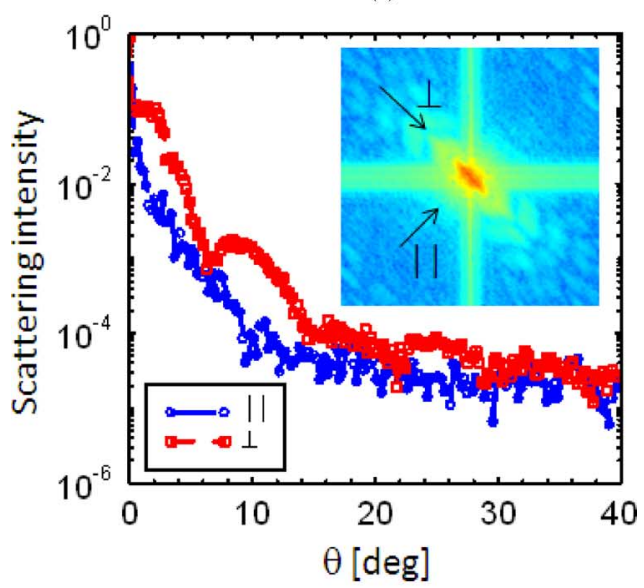

(b)

Fig. 4. (a) Quantitative phase image of a neuronal structure [67], [68]. The color bar indicates optical path length in nanometer $(\mathrm{nm})$ and the scale bar measures $10 \mu \mathrm{m}$. (b) Angular scattering associated with the putative axon selected by the dotted box in (a). The two profiles are along the perpendicular and parallel directions to the axon as indicated in the scattering map inset.

possibly the premise for a large scattering database, where various tissue types, healthy and diseased, will be fully characterized in terms of their scattering properties.

\section{FTLS OF CELL STRUCTURE}

Light scattering investigations can noninvasively reveal subtle details about the structural organization of cells [7], [8], [44]-[47]. We employed FTLS to measure scattering phase functions of different cell types and demonstrate its capability as a new modality for cell characterization. Fig. 4 shows an example of FTLS measurements from substructures of a live neuron, which, to our knowledge, cannot be attained using existing techniques. As shown in Fig. 4, FTLS has unique sensitivity to weak scatterers by the angular scattering from a putative axon.

In order to demonstrate the potential of FTLS for cell sorting based on the scattering signals that it provides, we retrieved the scattering phase functions from three cell groups [see Fig. 5(a)(c)]: red blood cells, myoblasts (C2C12), and neurons. Fig. 5(d) and (f) shows the angular scattering distributions associated with these samples. For each group, we performed measurements on different fields of view. Remarkably, FTLS provides
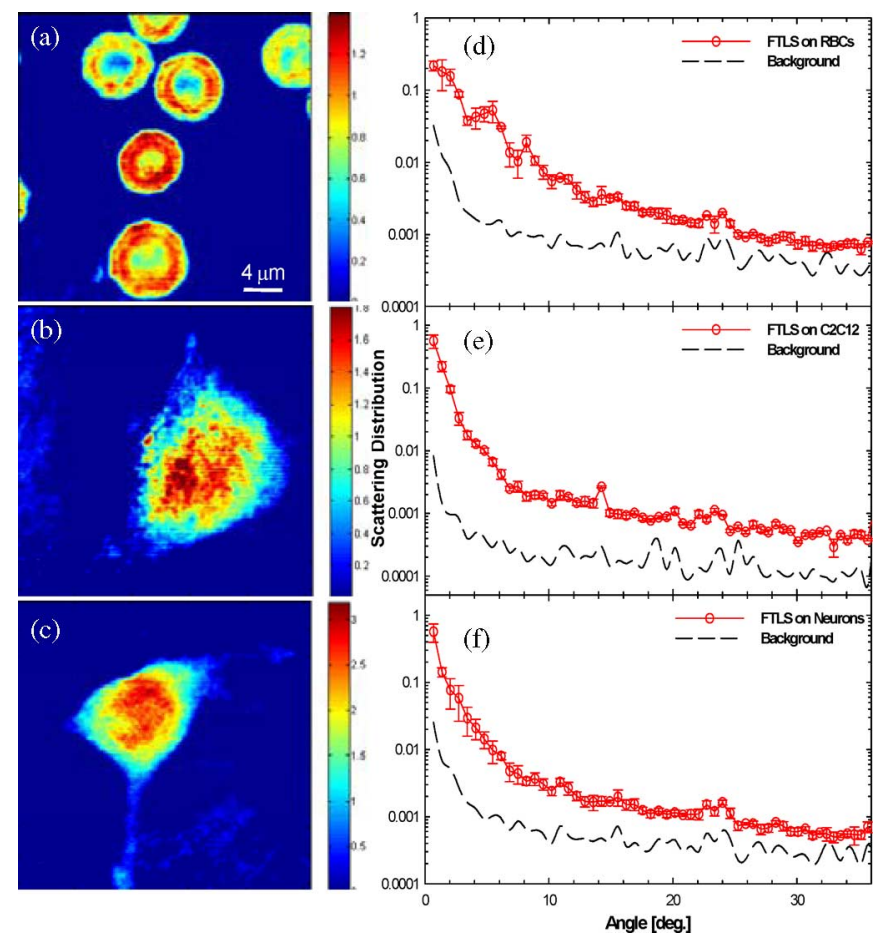

Fig. 5. (a) Quantitative phase images of red blood cells, (b) $\mathrm{C} 2 \mathrm{C} 12$ cell, and (c) neuron; the scale bar is $4 \mu \mathrm{m}$ and the color bar indicates phase shift in radians. (d)-(e) Respective scattering phase functions measured by FTLS

these scattering signals over approximately $35^{\circ}(40 \times$ objective applied for cell study) in scattering angle and several decades in intensity. For comparison, we also measured the scattering signature of the background (i.e., culture medium with no cells in the field of view by using threshold), which incorporates noise contributions from the beam inhomogeneities, impurities on optics, and residues in the culture medium. These measurements demonstrate that FTLS is sensitive to the scattering signals from single cells that contrast to previous measurements on cells in suspensions. Subtle details of the cell structures may be washed in studies on suspensions since the signals are averaged over various cell orientations.

We analyzed our FTLS data with a statistical algorithm based on the principle component analysis (PCA) aimed at maximizing the differences among the cell groups and providing an automatic means for cell sorting [48]. This statistical method mathematically transforms the data to a new coordinate system to illustrate the maximum variance by multiplying the data with the chosen individual vectors. Our procedure can be summarized as follows. First, we average the $n(n=1, \ldots, 45)$ measurements for the three cell types (15 measurements per group), to obtain the average scattered intensity, $\overline{I\left(\theta_{m}\right)}=(1 / 45) \sum_{n=1 . .45} I_{n}\left(\theta_{m}\right)$, with $m=1, \ldots, 35$ denoting the number of scattering angles. Second, we generate a matrix $\Delta Y_{\mathrm{nm}}$ of variances, where $n$ indexes the different measurements and $m$ the scattering angles. The covariance matrix associated with $\Delta Y, \operatorname{Cov}(\Delta Y)$, is calculated and its eigenvalues and eigenvectors extracted. The three principal components are obtained by retaining three eigenvectors corresponding to the largest eigenvalues. In order to build 

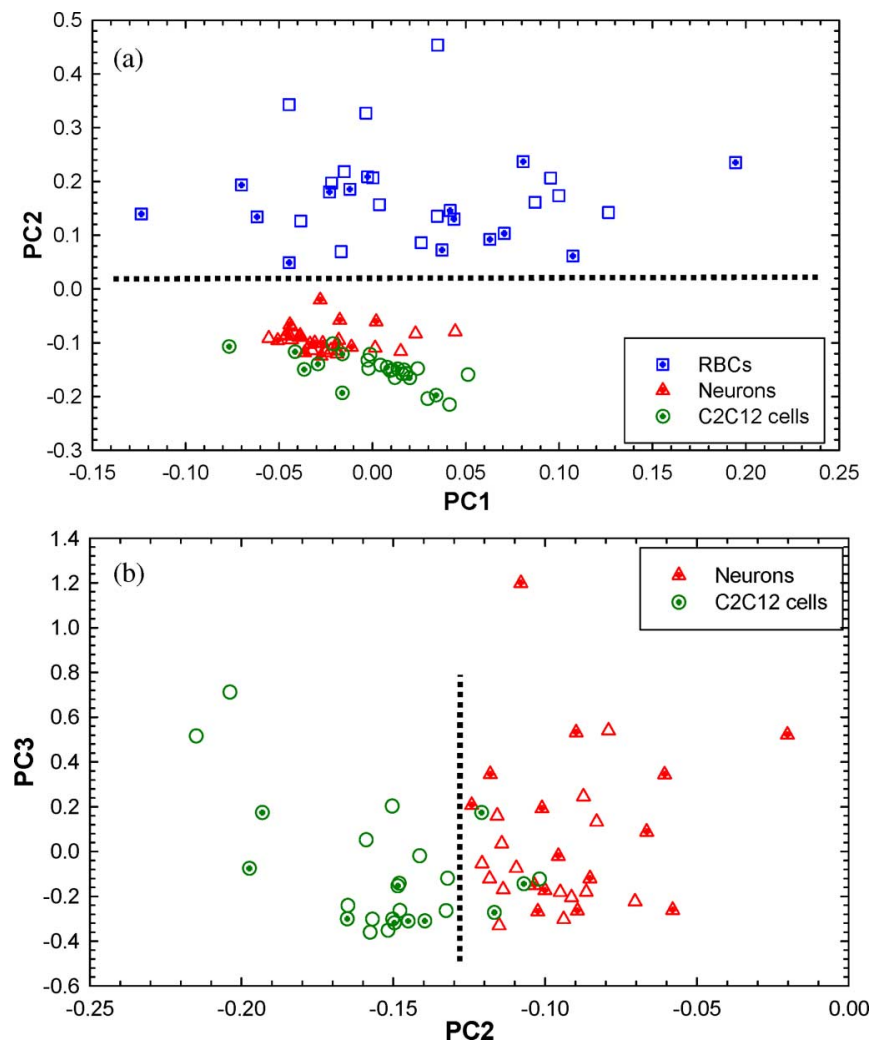

Fig. 6. PCA of the experimental data for the three cell types, as indicated. The symbols with "+" sign in the middle are the testing measurements for each sample, respectively. (a) PC2 versus PC1. (b) PC3 versus PC2.

the training set, 45 measurements (i.e., 15 per cell type) were taken and processed following the procedures described earlier.

Fig. 6(a) and (b) shows a representation of the data where each point in the plot is associated with a particular FTLS measurement. In addition to the 15 measurements per group for the training sets, we performed, respectively, 15,15 , and 10 test measurements for neurons, $\mathrm{RBCs}$, and $\mathrm{C} 2 \mathrm{C} 12$ cells. The additional test measurements allowed us to evaluate the sensitivity and specificity of assigning a given cell to the correct group [49]. We obtained sensitivity values of $100 \%, 100 \%$, and $70 \%$, and specificities of $100 \%, 88 \%$, and $100 \%$ for RBCs, neurons, and $\mathrm{C} 2 \mathrm{C} 12$ cells, respectively.

We demonstrated here that FTLS can be used to differentiate between various cell types. Due to the particular imaging geometry used, scattering phase functions associated with single cells can be retrieved over a broad range of angles. This remarkable sensitivity to weak scattering signals may set the basis for a new generation of cytometry technology, which, in addition to the intensity information, will extract the structural details encoded in the phase of the optical field. FTLS may improve on fluorescence-based flow cytometry as it operates without the need for exogenous tags.

\section{FTLS OF CELL DYNAMICS}

Recently, dynamic properties of cytoskeleton have been the subject of intense-scientific interest [50]-[60]. In particular, it
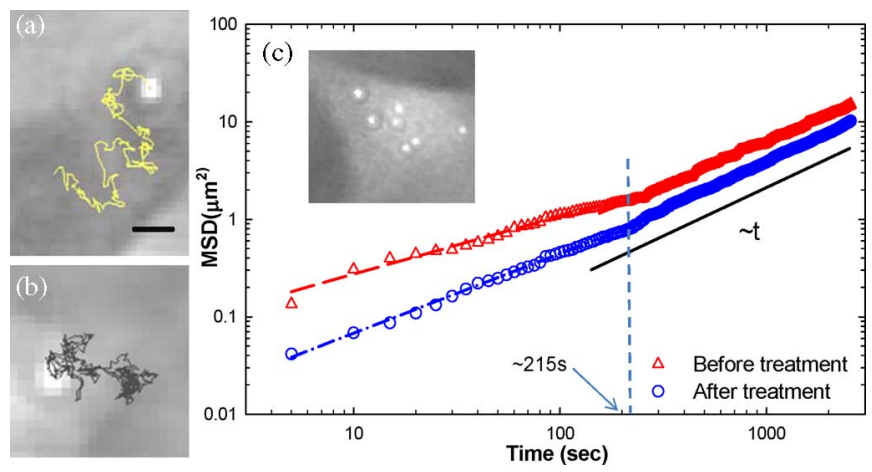

Fig. 7. Tracking beads attached to the cell membrane: (a) bead trajectories before Cyto-D treatment and (b) after drug treatment, scale bar for $2 \mu \mathrm{m}$. (c) Corresponding mean square displacement of the tracked beads before (red) and after (blue) treatment. The fitting with a power law function over two different time windows is indicated. The inset shows a quantitative phase image of an EGC with $1 \mu \mathrm{m}$ beads attached to the membrane.

has been shown that actin filaments play an important role in various aspects of cell dynamics, including cell motility [51]-[53]. Previously, actin polymerization has been studied in real time by total reflection fluorescence microscopy [54], [55]. In this section, we demonstrate that FTLS is capable of sensing the spatiotemporal behavior of active (ATP consuming) dynamics due to $f$-actin in single glial cells as addressed previously [61]. This activity is mediated by motor protein Myosin II and underlies diverse cellular processes, including cell division, developmental polarity, cell migration, filopodial extension, and intracellular transport.

We used FTLS to study the slow active dynamics of Enteric glial cytoskeleton. During the FTLS measurement, the cells were maintained under constant temperature at $37{ }^{\circ} \mathrm{C}$ via the incubation system that equips the microscope. The sensitivity of FTLS to actin dynamics was tested by controlling its polymerization activity. In order to inhibit actin polymerization, Cytochalasin-D (Cyto-D), approximately $5 \mu \mathrm{M}$ in Hibernate-A, was added to the sample dishes. Cyto-D is a naturally occurring fungal metabolite known to have potent inhibitory action on actin filaments by capping and preventing polymerization and depolymerization at the rapidly elongating end of the filament. By capping this "barbed" end, the increased dissociation at the pointed end continues to shorten the actin filament.

The established single-particle tracking method [22] was first applied to test the efficacy of Cyto-D as an actin inhibitor. With $1 \mu \mathrm{m}$ diameter beads attached to the cell membrane as probes, the dynamic property of the cell membrane was investigated. Sets of 512 quantitative phase images of cells with beads attached were acquired at 1 frame $/ 5 \mathrm{~s}$, with a total acquisition time of 45 minutes. The $x$ and $y$ coordinates of the tracked beads were recorded as a function of time and the trajectories were used to calculate the mean-squared displacement (MSD) [62]:

$$
M S D(\Delta t)=\left\langle[x(t+\Delta t)-x(t)]^{2}+[y(t+\Delta t)-y(t)]^{2}\right\rangle
$$

where $\langle\cdots\rangle$ indicates the average over time and also over all the tracked particles. We recorded the displacements of the attached beads before and after treatment with Cyto-D. The MSD results are summarized in Fig. 7. Each curve is the result of an average 

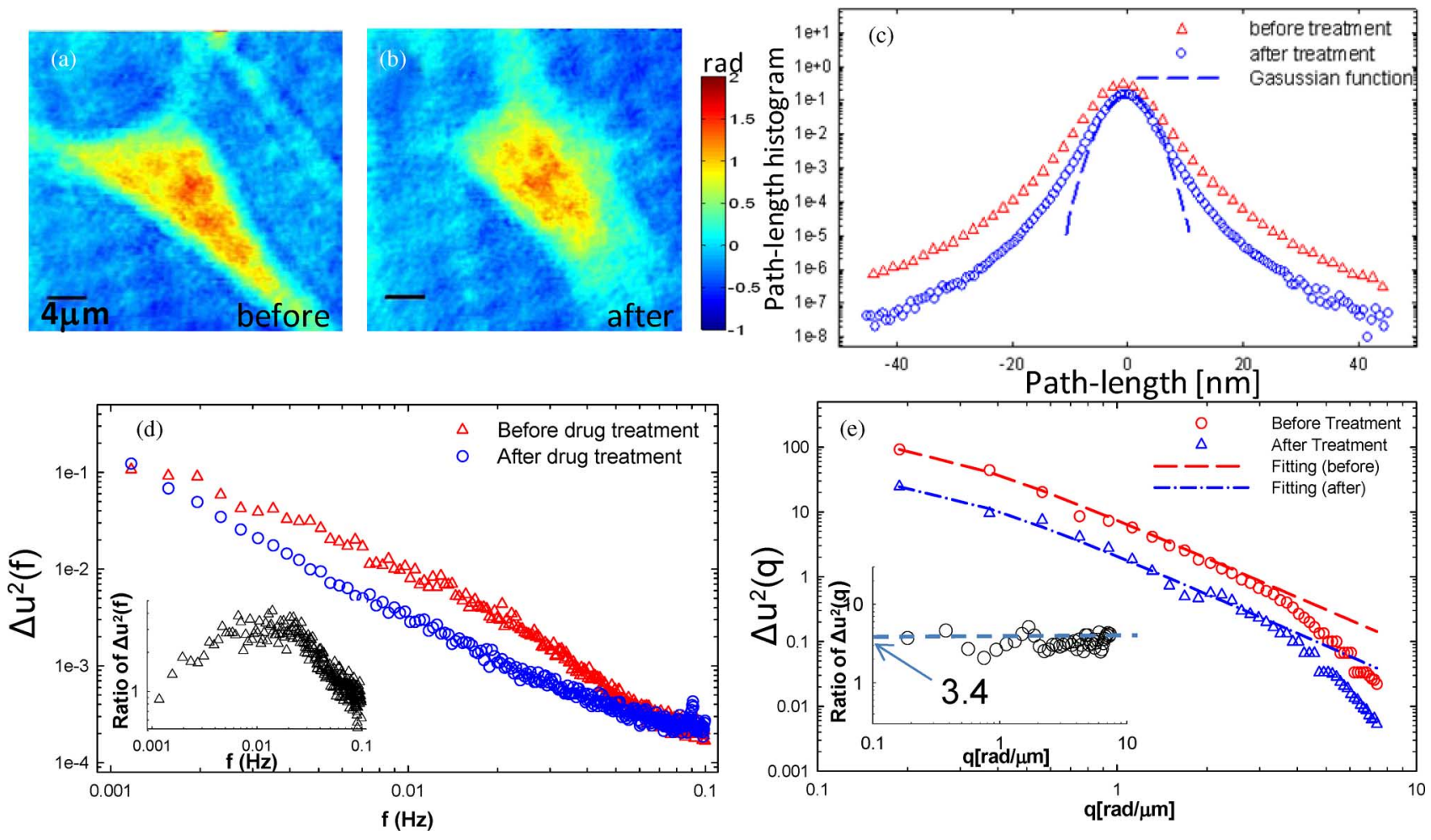

Fig. 8. (a) and (b) Quantitative phase images of glial cell before and after Cyto-D treatment. (c) Histogram of the path length displacements of a glial cell before and after drug treatment, as indicated. The blue dash line indicates the fit with a Gaussian function. (d) Spatially averaged power spectrum of glial cells before and after drug treatment, as indicated. The inset shows the ratio between these two power spectrums. (e) Temporally averaged power spectrum before and after drug treatment. The dash lines are the fittings with (7), and the inset shows the ratio of the two spectrums. Figure adapted from [61].

over all the beads $(N>10)$ tracked under the same experimental condition. The data for normal (before treatment) cells exhibit a power law trend over two distinct temporal regions, separated at approximately $\tau_{0}=215 \mathrm{~s}$. This change in slope reflects a characteristic lifetime $\tau_{0}$ associated with actin polymerization that is known to be in the minute range [63]. For both curves, the fit at $t>\tau_{0}$ gives a power law of exponent approximately 1, indicating diffusive motion. However, the membrane displacements for the untreated cells are a factor of 1.3 larger, consistent with the qualitative observation of the particle trajectories. Thus, at long times, the effect of actin on membrane dynamics is to enhance its motions without changing its temporal statistics.

To determine how the actin cytoskeleton contributes to the DLS of cells alone, cells without beads were imaged via DPM by acquiring 512 frames, at 0.2 frames per s, over $\sim 45 \mathrm{~min}$, prior to and after Cyto-D application, respectively [see Fig. 8(a) and (b)]. Fig. 8(c) shows a comparison between the membrane displacement histograms of a cell before and after the actin inhibitor. It is evident from this result that the polymerization phenomenon is a significant contributor to the overall cell membrane dynamics, as indicated by the broader histogram distribution. Further, both curves exhibit non-Gaussian shapes at displacements larger than $10 \mathrm{~nm}$, which suggests that the cell motions both before and after actin inhibition are characterized by nonequilibrium dynamics. Fig. 8(d) presents the comparison of the spatially averaged power spectra associated with the FTLS signal for a single cell, before and after treatment with the actin blocking drug. The broader power spectrum of the untreated cell mem- brane motions is consistent with the histogram distribution in Fig. 7(c) and also the particle tracking results. Further, both frequency-averaged (statics) curves shown in Fig. 8(e) indicate similar functional dependence on the wave vector $q$, but with enhanced fluctuations for the normal cell, by a factor of $\sim 3.4$. One key feature of FLTS is its ability to render simultaneously angular scattering from an entire range of angles. Fig. 9(a)(d) shows the power spectrum of the fluctuations for the same cell [as shown in Fig. 8(a) and (b)] before and after the actin inhibition, as function of both frequency $f$ (at two particular $q$ values) and wave vector $q$ (at two particular frequencies $\omega$ ). After actin inhibition, the functional dependence of $\Delta u^{2}(f)$ assumes a Lorentzian shape and does not change notably with varying $q$, which contrasts with the situation where the cell cytoskeleton is intact. This interesting behavior can be seen in Fig. 9(a) and (b), where the temporal power spectra at two particular scattering angles (or wave vectors) are shown. These findings suggest that as the actin is disrupted, the dynamic scattering signal is most likely due to Brownian-like membrane fluctuations. At frequencies $f>1 / \tau_{0}$ [see Fig. 9(c)], there is a significant mismatch between the "before" and "after" $\Delta u^{2}(q)$ curves. On the other hand, for $f<1 / \tau_{0}$ [see Fig. 9(d)], the two dependencies look similar, with the normal cell exhibiting consistently higher fluctuations.

The trends shown in Figs.7-9 support the idea of actinmediated membrane dynamics that is characterized by a time constant $\tau_{0}$. Thus, we propose a physical model which captures the essence of our experimental results. We assume that 

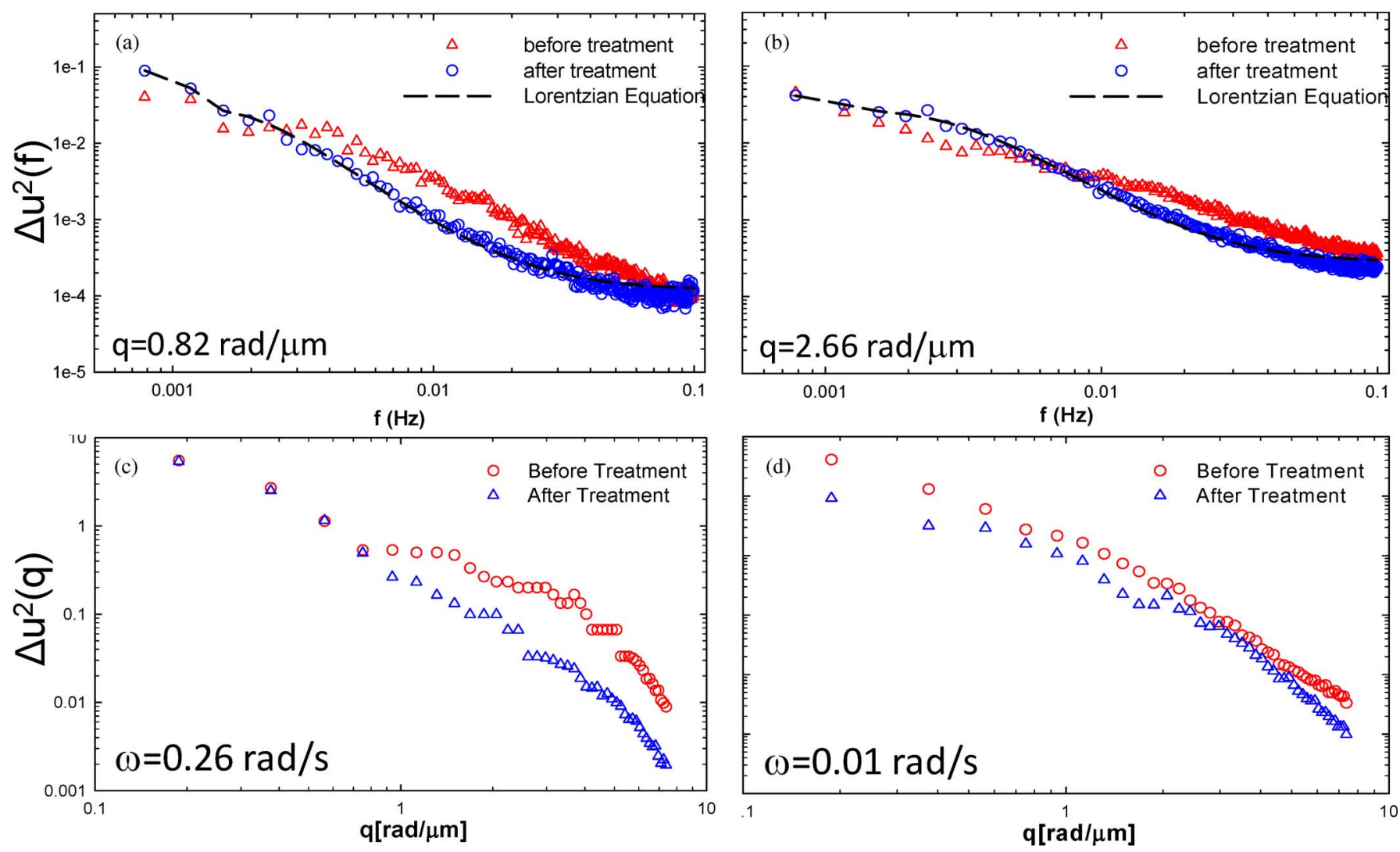

Fig. 9. (a) and (b) Temporal power spectrum of glial cells before and after drug treatment at two different spatial frequencies, as indicated. Dash lines show the fittings with Lorentzian equation. (c) and (d) Spatial power spectrum at two different frequencies which characterize cell dynamics at two distinct temporal regions, as indicated. Figure adapted from [61].

the cell membrane is under the influence of both Brownian motion and actively driven displacements due to ATP-consuming actin polymerization activity. The membrane is under tension and connected to springs (actin filaments) that undergo continuous remodeling. This picture has been applied successfully to the active motions in red blood cell membranes [64], [65]. The Langevin equation for the membrane fluctuations is [66]

$$
\dot{u}(q, t)+\omega(q) u(q, t)=\Lambda(q) f(q, t)
$$

where $u$ is the displacement of the membrane element, $q$ the spatial wave vector, $\omega(q)=\left(\kappa q^{3}+\sigma q+\gamma / q\right) / 4 \eta, \kappa$ the bending modulus, $\sigma$ the tension modulus, $\gamma$ the confinement factor, $\eta$ the viscosity of the surrounding medium, $f$ the random force, and $\Lambda(q)=1 /(4 \eta q)$ is the Oseen hydrodynamic factor. The displacement power spectrum can be obtained by Fourier transforming (10),

$$
|u(q, \omega)|^{2}=\frac{\Lambda^{2}(q)|f(q, \omega)|^{2}}{\omega^{2}+\omega(q)^{2}} .
$$

The instantaneous force has both Brownian and direct components, characterized by their respective temporal correlations,

$$
\begin{aligned}
\left\langle f_{B}(q, t) f_{B}(q, t+\tau)\right\rangle & =\frac{2 k_{B} T \delta(\tau)}{\Lambda(q)} \\
\left\langle f_{D}(q, t) f_{D}(q, t+\tau)\right\rangle & =\frac{2 \alpha k_{B} T\left(e^{-|\tau| / \tau_{0}} / 2 \tau_{0}\right)}{\Lambda(q)} .
\end{aligned}
$$

In (12) and (13), the $f_{B}$ is the Brownian (thermal) and $f_{D}$ the directed force, assumed to exhibit an exponential temporal correlation, with the decay time $\tau_{0}$ given by the actin polymerization lifetime. The angular brackets denote temporal average such that $\left\langle f_{B}\right\rangle=\left\langle f_{D}\right\rangle=0$. The factor $\alpha$ establishes the mean square force fluctuations, i.e., $\alpha=0$ for cyto-D treated cells. Combining (11)-(13), we obtain for the displacement power spectrum:

$$
|u(q, \omega)|^{2}=\frac{2 k_{B} T \Lambda(q)}{\omega^{2}+\omega(q)^{2}}\left[1+\alpha \frac{\omega_{0}^{2}}{\omega^{2}+\omega_{0}^{2}}\right] .
$$

Remarkably, this simple model captures some essential features of the experimental results, as follows. First, the active term in (14) captures the increase in the power spectrum at high frequencies [see Fig. 8(d)]. This is also evidenced in Fig. 9(c), where at a fixed (high) frequency, we found enhanced fluctuation spectrum versus $q$. Second, upon blocking actin polymerization with cyto-D, i.e., $\alpha \rightarrow 0,|u(q)|^{2}$ remains Lorentzian in shape for all $q$ 's, as found in Fig. 9(a) and (b). Third, at long times ( $\left.\omega \ll \omega_{0}\right)$, the active term is featureless, i.e., the actin contributions are Brownian-like, and can be described by an increased effective temperature $T_{\text {eff }}=\alpha T$ [65]. In this case, the static behavior is obtained integrating (14) over $\omega$, which yields

$$
|u(q)|^{2}=\frac{k_{B} T(1+\alpha)}{\left(\kappa q^{4}+\sigma q^{2}+\gamma\right)} .
$$


This explains the similar functional dependence for the two curves in Fig. 8(e), resulting in a ratio $\alpha+1=3.4$. A similar trend was found at a fixed (low) frequency, as shown in Fig. 9(d). We fitted the two curves in Fig. 8(e) with (15) and obtained a good agreement in the low $q$, tension-dominated region. The values for $\sigma$ obtained from the fit are $5.18 e-4$ and $1.85 e-3$, respectively. Note that both these values are approximately one to two orders of magnitude larger than those measured in red blood cells [33]. Thus, the membrane fluctuation noise produced by actin is equivalent to a decrease in surface tension or equivalently, an increase in effective temperature $T_{\text {eff }}=2.4 \mathrm{~T}$.

We believe that these initial results are extremely promising and the label-free approach proposed here for studying cytoskeleton dynamics will complement very well the existing fluorescence studies. However, the interpretation of these FTLS measurements requires improvement, as evidenced, for instance, in the poor fit of the curves in Fig. 8(e) at large $q$. It is likely that the simple model proposed here needs further refinements to include contributions from particle transport and polymer dynamics within the cell.

\section{Summary AND OUTLOOK}

In summary, we reviewed FTLS as a new approach for studying static and DLS with unprecedented sensitivity, granted by the image plane measurement of the optical phase and amplitude. In FTLS, spatial resolution of the scatterer positions is well-preserved. These remarkable features of FTLS are due to the interferometric experimental geometry and the reliable phase retrieval. FTLS has been applied to study the tissue optical properties, cell type characterization, and dynamic structure of cell membrane. We anticipate that this type of measurement will enable new advances in life sciences, due to the ability to detect weak scattering signals over broad temporal (milliseconds to days) and spatial (fraction of microns to centimeters) scales. Current efforts in our laboratory are focused on advancing the technique to study the mechanical properties of cells including the cell membrane tension, bending modulus, and 3-D shear modulus.

\section{ACKNOWLEDGMENT}

The authors thank Steve Boppart and the Biophotonics Imaging Laboratory for providing tissue samples, and the Network for Computational Nanotechonlogy, University of Illinois at Urbana-Champaign, for support.

\section{REFERENCES}

[1] R. Drezek, A. Dunn, and R. Richards-Kortum, "Light scattering from cells: Finite-difference time-domain simulations and goniometric measurements," Appl. Opt., vol. 38, no. 16, pp. 3651-3661, 1999.

[2] J. R. Mourant, M. Canpolat, C. Brocker, O. Esponda-Ramos, T. M. Johnson, A. Matanock, K. Stetter, and J. P. Freyer, "Light scattering from cells: The contribution of the nucleus and the effects of proliferative status," $J$. Biomed. Opt., vol. 5, no. 2, pp. 131-137, 2000.

[3] C. S. Mulvey, A. L. Curtis, S. K. Singh, and I. J. Bigio, "Elastic scattering spectroscopy as a diagnostic tool for apoptosis in cell cultures," IEEE J. Sel. Topics Quantum Electron., vol. 13, no. 6, pp. 1663-1670, Nov./Dec. 2007.
[4] H. Ding, J. Q. Lu, R. S. Brock, T. J. McConnell, J. F. Ojeda, K. M. Jacobs, and X. H. Hu, "Angle-resolved Mueller matrix study of light scattering by R-cells at three wavelengths of 442, 633, and $850 \mathrm{~nm}, "$ J. Biomed. Opt., vol. 12, no. 3, pp. 034032-1-034032-9, 2007.

[5] M. T. Valentine, A. K. Popp, D. A. Weitz, and P. D. Kaplan, "Microscopebased static light-scattering instrument," Opt. Lett., vol. 26, no. 12 , pp. 890-892, 2001.

[6] W. J. Cottrell, J. D. Wilson, and T. H. Foster, "Microscope enabling multimodality imaging, angle-resolved scattering, and scattering spectroscopy," Opt. Lett., vol. 32, no. 16, pp. 2348-2350, 2007.

[7] A. Wax, C. H. Yang, V. Backman, K. Badizadegan, C. W. Boone, R. R. Dasari, and M. S. Feld, "Cellular organization and substructure measured using angle-resolved low-coherence interferometry," Biophys. J., vol. 82, no. 4, pp. 2256-2264, 2002.

[8] V. Backman, M. B. Wallace, L. T. Perelman, J. T. Arendt, R. Gurjar, M. G. Muller, Q. Zhang, G. Zonios, E. Kline, T. McGillican, S. Shapshay, T. Valdez, K. Badizadegan, J. M. Crawford, M. Fitzmaurice, S. Kabani, H. S. Levin, M. Seiler, R. R. Dasari, I. Itzkan, J. Van Dam, and M. S. Feld, "Detection of preinvasive cancer cells," Nature, vol. 406, no. 6791, pp. 3536, 2000.

[9] D. F. Abbott, P. D. Kearney, and K. A. Nugent, "3-Dimensional imaging using an optical microscope," J. Mod. Opt., vol. 37, no. 11, pp. 1887-1893, 1990.

[10] B. J. Bern and R. Pecora, Dynamic Light Scattering With Applications to Chemistry, Biology and Physics. New York: Wiley, 1976.

[11] D. J. Pine, D. A. Weitz, P. M. Chaikin, and E. Herbolzheimer, "Diffusingwave spectroscopy," Phys. Rev. Lett., vol. 60, no. 12, pp. 1134-1137, 1988.

[12] M. Suissa, C. Place, E. Goillot, B. Berge, and E. Freyssingeas, "Dynamic light scattering as an investigating tool to study the global internal dynamics of a living cell nucleus," Epl-Europhys. Lett., vol. 78, no. 3, pp. 38005-1-38005-6, 2007.

[13] F. Charriere, N. Pavillon, T. Colomb, C. Depeursinge, T. J. Heger, E. A. D. Mitchell, P. Marquet, and B. Rappaz, "Living specimen tomography by digital holographic microscopy: Morphometry of testate amoeba," Opt. Exp., vol. 14, no. 16, pp. 7005-7013, 2006.

[14] D. Watson, N. Hagen, J. Diver, P. Marchand, and M. Chachisvilis, "Elastic light scattering from single cells: Orientational dynamics in optical trap," Biophys. J., vol. 87, no. 2, pp. 1298-1306, 2004.

[15] T. G. Mason and D. A. Weitz, "Optical measurements of frequencydependent linear viscoelastic moduli of complex fluids," Phys. Rev. Lett., vol. 74, no. 7, pp. 1250-1253, 1995.

[16] F. Gittes, B. Schnurr, P. D. Olmsted, F. C. MacKintosh, and C. F. Schmidt, "Microscopic viscoelasticity: Shear moduli of soft materials determined from thermal fluctuations," Phys. Rev. Lett., vol. 79, no. 17, pp. 32863289, 1997.

[17] V. Pelletier, N. Gal, P. Fournier, and M. L. Kilfoil, "Microrheology of microtubule solutions and actin-microtubule composite networks," Phys. Rev. Lett., vol. 102, no. 18, pp. 188303-1-188303-4, 2009.

[18] L. Ji, J. Lim, and G. Danuser, "Fluctuations of intracellular forces during cell protrusion," Nature Cell Biol., vol. 10, no. 12, pp. 1393-1400, 2008.

[19] D. Mizuno, C. Tardin, C. F. Schmidt, and F. C. MacKintosh, "Nonequilibrium mechanics of active cytoskeletal networks," Science, vol. 315, no. 5810, pp. 370-373, 2007.

[20] M. L. Gardel, M. T. Valentine, J. C. Crocker, A. R. Bausch, and D. A. Weitz, "Microrheology of entangled F-actin solutions," Phys. Rev. Lett., vol. 91, no. 15, pp. 158302-1-158302-4, 2003.

[21] J. C. Crocker, M. T. Valentine, E. R. Weeks, T. Gisler, P. D. Kaplan, A. G. Yodh, and D. A. Weitz, "Two-point microrheology of inhomogeneous soft materials," Phys. Rev. Lett., vol. 85, no. 4, pp. 888-891, 2000

[22] A. J. Levine and T. C. Lubensky, "One- and two-particle microrheology," Phys. Rev. Lett., vol. 85, no. 8, pp. 1774-1777, 2000.

[23] A. W. C. Lau, B. D. Hoffman, A. Davies, J. C. Crocker, and T. C. Lubensky, "Microrheology, stress fluctuations, and active behavior of living cells," Phys. Rev. Lett., vol. 91, no. 19, pp. 198101-1-198101-4, 2003.

[24] C. P. Brangwynne, G. H. Koenderink, E. Barry, Z. Dogic, F. C. MacKintosh, and D. A. Weitz, "Bending dynamics of fluctuating biopolymers probed by automated high-resolution filament tracking," Biophys. J., vol. 93, no. 1, pp. 346-359, 2007.

[25] M. S. Amin, Y. Park, N. Lue, R. R. Dasari, K. Badizadegan, M. S. Feld, and G. Popescu, "Microrheology of red blood cell membranes using dynamic scattering microscopy," Opt. Exp., vol. 15, no. 25, pp. 17001-17009, 2007. 
[26] A. Caspi, R. Granek, and M. Elbaum, "Diffusion and directed motion in cellular transport," Phys. Rev. E., vol. 66, no. 1, pp. 198101-1-198101-4, 2002.

[27] T. G. Mason, K. Ganesan, J. H. vanZanten, D. Wirtz, and S. C. Kuo, "Particle tracking microrheology of complex fluids," Phys. Rev. Lett., vol. 79, no. 17, pp. 3282-3285, 1997.

[28] N. Lue, J. Bewersdorf, M. D. Lessard, K. Badizadegan, K. Dasari, M. S. Feld, and G. Popescu, "Tissue refractometry using Hilbert phase microscopy," Opt. Lett., vol. 32, pp. 3522-3524, 2007.

[29] B. Rappaz, P. Marquet, E. Cuche, Y. Emery, C. Depeursinge, and P. J. Magistretti, "Measurement of the integral refractive index and dynamic cell morphometry of living cells with digital holographic microscopy," Opt. Exp., vol. 13, no. 23, pp. 9361-9373, 2005.

[30] W. Choi, C. C. Yu, C. Fang-Yen, K. Badizadegan, R. R. Dasari, and M. S. Feld, "Feild-based angle-resolved light-scattering study of single live COS," Opt. Lett., vol. 33, no. 14, pp. 1596-1598, 2008.

[31] G. Popescu, T. Ikeda, R. R. Dasari, and M. S. Feld, "Diffraction phase microscopy for quantifying cell structure and dynamics," Opt. Lett., vol. 31, no. 6, pp. 775-777, 2006.

[32] H. F. Ding, Z. Wang, F. Nguyen, S. A. Boppart, and G. Popescu, "Fourier transform light scattering of inhomogeneous and dynamic structures," Phys. Rev. Lett., vol. 101, no. 23, pp. 238102-1-238102-4, 2008.

[33] G. Popescu, T. Ikeda, K. Goda, C. A. Best-Popescu, M. Laposata, S. Manley, R. R. Dasari, K. Badizadegan, and M. S. Feld, "Optical measurement of cell membrane tension," Phys. Rev. Lett., vol. 97, pp. 218101-1218101-4, 2006.

[34] T. Ikeda, G. Popescu, R. R. Dasari, and M. S. Feld, "Hilbert phase microscopy for investigating fast dynamics in transparent systems," Opt. Lett., vol. 30, no. 10, pp. 1165-1168, 2005.

[35] H. C. van de Hulst, Light Scattering by Small Particles. New York: Dover, 1981.

[36] V. V. Tuchin, Tissue Optics. Bellingham, WA: SPIE-The International Society for Optical Engineering, 2000.

[37] J. W. Pyhtila, K. J. Chalut, J. D. Boyer, J. Keener, T. D’Amico, M. Gottfried, F. Gress, and A. Wax, "In situ detection of nuclear atypia in Barrett's esophagus by using angle-resolved low-coherence interferometry," Gastrointest. Endosc., vol. 65, no. 3, pp. 487-491, 2007.

[38] H. Fang, L. Qiu, E. Vitkin, M. M. Zaman, C. Andersson, S. Salahuddin, L. M. Kimerer, P. B. Cipolloni, M. D. Modell, B. S. Turner, S. E. Keates, I. Bigio, I. Itzkan, S. D. Freedman, R. Bansil, E. B. Hanlon, and L. T. Perelman, "Confocal light absorption and scattering spectroscopic microscopy," Appl. Opt., vol. 46, no. 10, pp. 1760-1769, 2007.

[39] H. Ding, F. Nguyen, S. A. Boppart, and G. Popescu, "Optical properties of tissues quantified by Fourier transform light scattering," Opt. Lett., vol. 34, pp. 1372-1374, 2009.

[40] G. Popescu, "Quantitative phase imaging of nanoscale cell structure and dynamics," in Methods in Cell Biology, P. J. Bhanu, Ed.. Amsterdam, The Netherlands: Elsevier, 2008, pp. 87-115.

[41] L. O. Reynolds and N. J. Mccormick, "Approximate 2-parameter phase function for light-scattering," J. Opt. Soc. Amer, vol. 70, no. 10, pp. 12061212,1980

[42] J. M. Schmitt and G. Kumar, "Optical scattering properties of soft tissue: A discrete particle model," Appl. Opt., vol. 37, no. 13, pp. 2788-2797, 1998.

[43] M. S. Patterson, B. Chance, and B. C. Wilson, "Time resolved reflectance and transmittance for the noninvasive measurement of tissue opticalproperties," Appl. Opt., vol. 28, no. 12, pp. 2331-2336, 1989.

[44] J. D. Wilson and T. H. Foster, "Characterization of lysosomal contribution to whole-cell light scattering by organelle ablation," J. Biomed. Opt., vol. 12, no. 3, pp. 030503-1-030503-3, 2007.

[45] J. R. Mourant, J. P. Freyer, A. H. Hielscher, A. A. Eick, D. Shen, and T. M. Johnson, "Mechanisms of light scattering from biological cells relevant to noninvasive optical-tissue diagnostics," Appl. Opt., vol. 37, no. 16 , pp. 3586-3593, 1998.

[46] G. Popescu and A. Dogariu, "Scattering of low coherence radiation and applications," Eur. Phys. J., vol. 32, no. 2, pp. 73-93, 2005 (Invited Review Paper).

[47] S. A. Alexandrov, T. R. Hillman, and D. D. Sampson, "Spatially resolved Fourier holographic light scattering angular spectroscopy," Opt. Lett., vol. 30 , no. 24 , pp. 3305-3307, 2005.

[48] I. T. Jolliffe, Principal Component Analysis, 2nd ed ed. New York: Springer-Verlag, 2002.

[49] T. W. Loong, "Understanding sensitivity and specificity with the right side of the brain," Br. Med. J., vol. 327, no. 7417, pp. 716-719, 2003.
[50] M. L. Gardel, J. H. Shin, F. C. MacKintosh, L. Mahadevan, P. Matsudaira, and D. A. Weitz, "Elastic Behavior of cross-linked and bundled actin networks," Science, vol. 304, no. 5675, pp. 1301-1305, 2004.

[51] T. D. Pollard and G. G. Borisy, "Cellular motility driven by assembly and disassembly of actin filaments," Cell, vol. 112, no. 4, pp. 453-465, 2003.

[52] J. A. Cooper and D. A. Schafer, "Control of actin assembly and disassembly at filament ends," Curr. Opin. Cell Biol., vol. 12, no. 1, pp. 97-103, 2000.

[53] T. J. Mitchison and L. P. Cramer, "Actin-based cell motility and cell locomotion," Cell, vol. 84, no. 3, pp. 371-379, 1996.

[54] J. R. Kuhn and T. D. Pollard, "Real-time measurements of actin filament polymerization by total internal reflection fluorescence microscopy," Biophys. J., vol. 88, no. 2, pp. 1387-1402, 2005.

[55] T. D. Pollard, "The cytoskeleton, cellular motility and the reductionist agenda," Nature, vol. 422, no. 6933, pp. 741-745, 2003.

[56] K. J. Amann and T. D. Pollard, "Direct real-time observation of actin filament branching mediated by Arp $2 / 3$ complex using total internal reflection fluorescence microscopy," Proc. Natl. Acad. Sci. USA, vol. 98, no. 26, pp. 15009-15013, 2001.

[57] J. A. Theriot and T. J. Mitchison, "Actin microfilament dynamics in locomoting cells," Nature, vol. 352, no. 6331, pp. 126-131, 1991.

[58] J. A. Theriot, T. J. Mitchison, L. G. Tilney, and D. A. Portnoy, "The rate of actin-based motility of intracellular listeria-monocytogenes equals the rate of actin polymerization," Nature, vol. 357, no. 6375, pp. 257-260, 1992.

[59] D. Uttenweiler, C. Veigel, R. Steubing, C. Gotz, S. Mann, H. Haussecker, B. Jahne, and R. H. A. Fink, "Motion determination in actin filament fluorescence images with a spatio-temporal orientation analysis method," Biophys. J., vol. 78, no. 5, pp. 2709-2715, 2000.

[60] C. C. Wang, J. Y. Lin, H. C. Chen, and C. H. Lee, "Dynamics of cell membranes and the underlying cytoskeletons observed by noninterferometric widefield optical profilometry and fluorescence microscopy," Opt. Lett., vol. 31, no. 19, pp. 2873-2875, 2006.

[61] L. J. M. H. Ding, M. U. Gillette, and G. Popescu, "Spatio-temporal cytoskeleton fluctuations probed by Fourier transform light scattering," Phys. Rev. Lett., to be published.

[62] P. Dieterich, R. Klages, R. Preuss, and A. Schwab, "Anomalous dynamics of cell migration," Proc. Natl. Acad. Sci. USA, vol. 105, no. 2, pp. 459 463, 2008.

[63] N. Watanabe and T. J. Mitchison, "Single-molecule speckle analysis of actin filament turnover in lamellipodia," Science, vol. 295, no. 5557, pp. 1083-1086, 2002.

[64] L. C. L. Lin, N. Gov, and F. L. H. Brown, "Nonequilibrium membrane fluctuations driven by active proteins," J. Chem. Phys., vol. 124, no. 7, pp. 074903-1-074903-15, 2006.

[65] N. Gov, "Membrane undulations driven by force fluctuations of active proteins," Phys. Rev. Lett., vol. 93, no. 26, pp. 268104-1-1-268104-1-4, 2004.

[66] G. Popescu, Y. K. Park, R. R. Dasari, K. Badizadegan, and M. S. Feld, "Coherence properties of red blood cell membrane motions," Phys. Rev. E., vol. 76, pp. 031902-1-031902-5, 2007.

[67] Z. Wang and G. Popescu, "Quantitative phase imaging with broadband fields," Appl. Phy. Lett., vol. 96, 051117, 2010.

[68] Z. Wang, L. Millet, H. Ding, M. Mir, S. Unarunotai, J. Rogers, M. Gillette, and G. Popescu, "Spatial Light Interference Microscope (SLIM)," Nature Methods, submitted for publication.

Huafeng Ding received the B.Sc. degree in applied physics from Nankai University, Tianjin, China, in 2000, and the Ph.D. degree in biomedical physics from East Carolina University, Greenville, NC, in 2006.

$\mathrm{He}$ is currently a Postdoctoral Research Associate with Beckman Institute for Advanced Science and Technology, Department of Electrical and Computer Engineering, University of Illinois at Urbana-Champaign, Urbana. He is currently engaged in the application of the Fourier transform light scattering to study the structure and dynamic activity of biological tissues and live cells. His current research interests include the development of new optical techniques to study biological tissues and cells and also in application of those novel tools to early diagnosis of disease or treatment planning. 
Ethan Berl graduated from the University Laboratory High School, Urbana, IL, in 2008.

$\mathrm{He}$ is currently with the Computer Science Department, Princeton University, Princeton, NJ. During 2009, under the generous funding from the Network for Computational Nanotechonlogy at Illinois, he was engaged to develop Java programs built on the ImageJ platform for the mathematical toolbox of the Quantitative Light Imaging Laboratory, Beckman Institute for Advanced Science and Technology, University of Illinois at Urbana-Champaign, Urbana. These programs make it possible to locate and analyze cell behaviors in very large sets of image data in both the spatial and frequency domains. His current research interests include various methods of machine learning and image processing techniques.

Zhuo Wang received the B.Sc. degree in precision instruments from Tsinghua University, Beijing, China, in 2002, and the Master's degree in electrical engineering from Wayne State University, Detroit, MI, in 2006.

$\mathrm{He}$ is currently with the Department of Electrical and Computer Engineering, University of Illinois at Urbana-Champaign, Urbana. His current research interests include development of optical system for the study of biological tissues and live cells, and also the application to clinic.

Larry J. Millet received the B.S. degree in biology from Portland State University, Portland, OR, and the Ph.D. degree in cell and developmental biology in 2009 from the University of Illinois at Urbana-Champaign, Urbana, where he is currently a Postdoctoral Research Associate in micro and nanotechnology.

His current research interests include combining developmental biology and neurobiology with engineering disciplines for improved insight into the structure and function of cells.

Martha U. Gillette received the B.A. degree in biology from Grinnell College, Grinnell, IA, in 1969, and the Ph.D. degree in developmental biology from the University of Toronto, Toronto, ON, Canada, in 1976.

She was a Postdoctoral in neuroscience at the University of California, Santa Cruz. She joined the faculty at the University of Illinois, where she is Center for Advanced Study Professor, Alumni Professor of cell and developmental biology, and holds additional appointments in physiology, neuroscience, and bioengineering. Her current research interests include to understand the actions of actin in neurons and glia, signal processing in brain, and the consequences for what animals do. Approaches combine cellular neuroscience, nanoscale analytical chemistry, nanoengineering, and noninvasive imaging.
Jianming Liu received the B.Sc. degree in biochemistry and molecular biology from Nankai University, Tianjin, China, in 2002, and the Ph.D. degree in cell and developmental biology in 2007 from the University of Illinois a Urbana-Champaign, Urbana, where he was a Postdoctoral Research Associate in Beckman Institute for Advanced Science and Technology, Department of Kinesiology and Community Health.

He is currently a Postdoctoral Fellow in the Department of Cell and tissue Biology, University of California, San Francisco. His current research interests include to study muscle stem cells differentiation and skeletal muscle development and physiology.

Marni Boppart received the Bachelor's degree in molecular, cellular and developmental biology from the University of New Hampshire, Durham, in 1992, the Master's degree in cell biology from Creighton University, Omaha, NE, in 1996, and the Sc.D. degree in applied anatomy and physiology from Boston University, Boston, MA, in 2000.

She was an Officer and the Aerospace Physiologist in the U.S. Air Force. She was with the Joslin Diabetes Center, Harvard Medical School. She was a Postdoctoral in the Department of Cell and Developmental Biology, University of Illinois at Urbana-Champaign, Urbana, where she is currently an Assistant Professor in the Department of Kinesiology and Community Health and is a Full-Time Faculty in the Beckman Institute for Advanced Science and Technology. Her current research interests include cellular biomechanics, cell signaling, and the role of extracellular matrix proteins in the protection of skeletal muscle from injury, disease, and aging.

Gabriel Popescu received the B.S. and M.S. degrees in physics from the University of Bucharest, Bucharest, Romania, in 1995 and 1996, respectively, and the M.S. and Ph.D. degrees in optics from the School of Optics/CREOL (now the College of Optics and Photonics), University of Central Florida, Orlando, in 1999 and 2002, respectively.

In 2002, he became a Postdoctoral Associate in the G. R. Harrison Spectroscopy Laboratory, Massachusetts Institute of Technology (MIT). In August 2007, he joined the University of Illinois at Urbana-Champaign (UIUC), Urbana, where he is currently an Assistant Professor in the Department of Electrical and Computer Engineering, holds a Full Faculty appointment with the Beckman Institute for Advance Science and Technology, and is also an Affiliate Faculty in the Bioengineering Department. 